\title{
García Manrique, Ricardo. Se vende cuerpo. El debate sobre la venta de órganos. Barcelona: Herder, 2021.
}

Lukas Romero-Wenz

Universitat de València

Fecha de recepción 11/10/2021 I De publicación: 23/12/2021

Ricardo García Manrique, catedrático de Filosofía del Derecho de la Universitat de Barcelona, acaba de publicar un libro abordando una problemática del tipo que suelen generar debate en entornos de reflexión política y jurídica. Se trata de la cuestión de la venta de órganos, en cuya argumentación, a favor o en contra, intervienen concepciones distintas sobre la libertad y dignidad humanas, y sobre los límites de la mercantilización.

En este libro, asumiendo como punto de partida un mercado de órganos lo más justo posible (entro en ello unas líneas más abajo), se hace un análisis de los distintos argumentos que pudieran interponerse a dicha práctica, para acabar calificando a la venta de órganos como una "actividad de alto riesgo", que conviene no introducir en una sociedad por sus corrosivos efectos.

Un primer aspecto a destacar de esta obra es su claridad expositiva: el autor conduce su análisis cubriendo cada uno de los aspectos en cada punto de su argumentación. Expone presentando, para desglosar en posibles aspectos (distintas características, o bien diferentes objeciones, o variados argumentos con los que se defiende una objeción, etc.), abordándolos después de uno en uno (o bien explicando por qué se ha desechado metodológicamente entrar en ciertos temas). Esto resulta en un texto que, pese a un rigor argumentativo que no rehúye entrar en profundidades, resulta muy fácil de seguir para el lector medio. El autor dibuja con talento un esquema arbóreo en la mente del lector, que no tiene en ningún momento sensación de "estar perdido" en la explicación. Cuestión nada fácil, como sabrá cualquiera que se haya dedicado a la docencia y/o la escritura.

El libro inicia aceptando que una propuesta de mercado de órganos pueda resultar razonable: ese mercado aumentaría su oferta, de forma que personas que mueren esperando trasplante llegarían a recibir uno. Siguiendo la propuesta de Erin y Harris, Manrique describe un mercado de órganos ético con las 
siguientes características: 1) el comprador sería uno solo, y sería una institución pública; 2) se circunscribiría políticamente a un Estado o grupo de ellos (v.gr. la UE), y sólo sus ciudadanos podrían comprar o vender; 3 ) el precio de compra sería único y fijado por el comprador público; 4) la distribución de los órganos no se haría por criterios mercantiles sino en base a una concepción justa de la prioridad médica. El autor explica que así se desactivan algunos argumentos en relación con las posibles prácticas de explotación, al regular el acceso al mercado, el precio y la distribución.

Hay cuatro argumentos por los que se aduce la legitimidad de un mercado de órganos así considerado: primero, porque salvaría vidas y mejoraría la salud. Segundo, por una cuestión de respeto a la propiedad, entendiendo la relación con el propio cuerpo como una propiedad de sí mismo, de la que cada sujeto podría disponer. Manrique señala varias debilidades en las ideas básicas de este argumento, rechazándolo ya en la exposición preliminar. Tercero, se argumenta la autonomía individual, es decir, el hecho de que no se debería impedir un uso de la libertad que no afecte a terceros. Cuarto, se realiza una petición de coherencia. Si se permiten otras actividades de algún modo similares: peligrosas (escalar montañas, por ejemplo), o que impliquen "venta de uno mismo" (trabajar vendiendo el propio tiempo y esfuerzo), ¿por qué no ésta? Nuestro autor vuelve a mostrar algunos fallos en el argumento, y a señalar que en realidad es una versión del cuestionamiento tercero, que habrá de responder.

Es decir, los argumentos que se señalan son un aumento de la salud/ posibilidad de salvar vidas, y la cuestión de la autonomía: la venta de órganos, siendo beneficiosa y estando regulada para prevenir la explotación, quedaría como una actividad que no habría razones para prevenir.

García Manrique, sin embargo, señala dos argumentos que objetan esta visión: el argumento "de la desigualdad" y el “de la degradación”. Prácticamente todo el cuerpo central del libro se ocupa de analizar y discutir estos dos argumentos, con especial atención al segundo.

Los grupos de argumentos "de desigualdad" insisten en que son las personas con rentas más bajas las que, previsiblemente, se verían forzadas a dicha actividad: el mercado de órganos se apoyaría en una falta de igualdad que contribuiría además a exacerbar. Se argumenta que no hay verdadero consentimiento, o que hay coacción, por la mala posición en que está una de las partes, que prácticamente se vería forzada a vender el órgano. Se argumenta asimismo que se da una explotación, aunque esta puede entenderse de dos maneras: explotación porque se trata "como medio y no como fin" al vendedor (en cuyo caso este argumento pertenecería al grupo de argumentos que nuestro autor llama "de la 
degradación", por basarse en la dignidad humana) o bien, a modelo del análisis marxista del trabajo, porque una parte resulta muy beneficiada por el gran sacrificio que efectúa la otra.

Nuestro autor analiza tres tipos de argumentos "de la desigualdad", explicando por qué le resultan descartables, y justificando así su mayor atención al otro argumento. El primer tipo expone que en el intercambio mercantil la posición de las dos partes es desigual, lo que lleva a concluir su injusticia. García Manrique señala que esto es en realidad una crítica al mismo mercado, en el que lo difícil es encontrar situaciones de completa igualdad. El argumento, así, se vuelve trivial en un debate que no problematiza la existencia del mercado en general. Un segundo tipo de argumentos señala que la desigualdad lo sería por razón del precio, injustamente bajo. Pero, responde Manrique, esto no podría ser un argumento en contra de todo el mercado de órganos, sino solo de uno que no asegurase un precio adecuado. Se podrían imaginar maneras de intervenir el mercado para ajustar sus precios, como la propuesta de mercado que plantean Erin y Harris. Por último, se argumenta la desigualdad por la naturaleza específica de lo corporal, y eso depende por entero de la consideración que se tenga del cuerpo como algo dotado de una dignidad especial. Es decir, este tercer argumento formaría en realidad parte del cuerpo de argumentos "de la degradación".

Expuesto el argumento de la desigualdad, el autor pasa a fijarse en el argumento "de la degradación". Empieza exponiéndolo y analizándolo. Respecto a su exposición, lo describe con el silogismo: "Las prácticas (especialmente) degradantes no deben ser permitidas por el derecho, y la venta de órganos es una práctica de ese tipo; por tanto, el derecho no debe permitirla". El autor señala la conveniencia de incluir el "especialmente" en la formulación del argumento, por cuanto que el derecho no debería sustituir el papel de la moral impidiendo toda práctica que pudiera ser considerada degradante. Y señala que lo máximo que se puede hacer para una argumentación útil es aducir razones varias que, en concurrencia, justifiquen la prohibición, ya que la objetivación última de lo "especialmente degradante" no parece posible, dada la tendencia de ese tipo de discusiones a devenir en disquisiciones interminables.

Respecto al análisis, señala varias cuestiones en torno al argumento: la primera es remarcar el hecho de que la concurrencia de lo corporal y lo monetario parece fundamental para juzgar como degradante la actividad (caso parecido al de la prostitución y la maternidad subrogada comercial). Segunda, se realiza la pregunta por la práctica degradante, llegando a la conclusión de que tal adjetivo solo es aplicable con el concepto de "dignidad" de fondo, y que, dados los distintos conceptos de "dignidad" que parecen entrecruzarse en las discusiones (apoyados en diferentes visiones del muy relacionado concepto de 
“libertad”), habrá que clarificar cómo se entiende esa "dignidad” (cosa que hará más adelante). Tercera, se pregunta acerca de si sería mejor la opción de "prohibir" frente a la de “desalentar". García Manrique señala su objetivo de despejar la pregunta por el carácter degradante de la venta, no mostrándose seguro respecto a qué instrumento jurídico se hubiera de utilizar si dicho carácter se demostrara. Cuarta, se hace la pregunta por el carácter corruptor del dinero, señalando que toca responder a la pregunta de si dos prácticas legítimas por separado (donar un órgano y comerciar) pueden ser consideradas degradantes al combinarse. Y a esa cuestión añade además otras dos, recapituladas de sus consideraciones anteriores: la de si tal degradación es tan grave como para justificar la intervención del derecho, y la de si el medio adecuado para tal intervención es la prohibición.

Analizado el argumento en su versión fundamental, entra García Manrique en el apartado más profundo del libro: la exposición de dos versiones del argumento de la degradación, una individual, fuertemente apoyada en Kant, y otra social o comunitaria.

Respecto a su versión individual, el autor expone el argumento kantiano acerca de la dignidad. Recoge el imperativo categórico en su segunda formulación, que exige tratar a la persona siempre como fin, y nunca como medio. La persona no tiene precio, por cuanto que tiene dignidad. A la persona no le sería lícito, según Kant, disponer de sí misma vendiendo una parte de su cuerpo, en base a esa misma dignidad, por cuanto que algo así sería "suicidio parcial”. Muy resumidamente: García Manrique acepta que se puede construir un argumento "de tipo kantiano" sobre la venta de las partes del cuerpo, es decir, un argumento basado en la idea de dignidad del filósofo prusiano, si bien no le convence el argumento usado por Kant específicamente (la comparativa con el suicidio).

Pero para dar por válido ese argumento, García Manrique entra a analizar el estatus del cuerpo, qué consideramos que es el cuerpo. Realiza esto en un capítulo aparte, siguiente a aquel donde ha expuesto el concepto de dignidad kantiano. Ahí, analiza la complicación de la consideración del cuerpo, por cuanto que sí que puede verse en cierto sentido relativo como "cosa" (si no, tampoco sería lícita la donación de órganos, o de sangre). Eso obliga a pensar su estatus, marcando los límites a esa capacidad de extrañamiento parcial. Repasa varias cuestiones en torno a esto, como, por ejemplo, la consideración que hay que tener del órgano separado del cuerpo, por cuanto que no es algo susceptible de ser usado de cualquier manera, pero tampoco es "una persona". Otro aspecto que también aborda es la consideración que tenemos respecto a nuestro cuerpo y si el cambio científico y tecnológico puede influir en ella; y asimismo se pregunta si un cambio por esas razones sería necesariamente malo; entre otras cuestiones. 
Su conclusión en el capítulo es que, si bien no se puede decir que las partes del cuerpo sean "fines en sí mismas" o "posean dignidad", sí participan en la naturaleza corporal humana, que es a su vez asimilable a la persona humana. El cuerpo humano, también sus órganos, estarían en el lado de lo que Kant consideraba sujeto de dignidad y no de precio, y por tanto su venta o compra es un atentado contra la dignidad propia (al vender) o ajena (al comprar), es decir, una práctica degradante.

Respecto al argumento de la degradación desde el punto de vista comunitario, el autor inicia recogiendo la discusión a partir del análisis que realizara Titmuss entre el modelo británico y el estadounidense de donación de sangre, siendo el estadounidense uno en que se pagaba al donante y el británico uno en el que la donación era puramente altruista. En dicho debate, las cuestiones que se plantean giran en torno a la preocupación por proteger la práctica social del altruismo, que un sistema de precios podría dinamitar (es decir, que al poner precio a la donación, dicho precio no tiene solo el efecto de unir al caudal de los "donantes gratuitos" el de los "donantes incentivados", sino que eliminaría parcial o totalmente ese primer caudal). El capítulo gira en torno a la cuestión del papel social del altruismo, y el cambio social potencialmente dañino que puede generar la comercialización, por destruir ese altruismo, por cambiar la percepción de los individuos sobre sus cuerpos, y varias cuestiones más. Las conclusiones del autor a este respecto son que un argumento en contra de la donación de órganos por la especialidad del cuerpo humano podría articularse construyéndose en torno a estas razones, que han de entenderse interconectadas: 1) la donación de órganos es una práctica altruista; 2) abrir el mercado reduciría el altruismo en la sociedad, lo cual siempre es una mala noticia a nivel social; 3) esa reducción del altruismo no sería cualquiera, sino una que afectaría al cuerpo humano, que no parece adecuado considerar similar a cualquier otro bien por cuanto que afecta al "estar en el mundo" de la persona; 4) la sacralidad e inviolabilidad del cuerpo; 5) la exigencia de respeto al cuerpo nacida de la conciencia de sacralidad del mismo; 6) la especialidad del altruismo implicado en la donación de órganos, resultante de la especialidad del cuerpo humano; 7) la inconveniencia de marcar con un precio los órganos, por el entendimiento de los mismos como mercancía fungible que pudiera resultar, indigna si tenemos en cuenta su especial estatus como partes del cuerpo; 8) la necesidad, por tanto, de una conciencia especial del cuerpo; 9) la argumentación de la degradación, por tanto, que supondría la posibilidad de disponer de los propios órganos como mercancías intercambiables por precio.

Tras esta doble aproximación, el autor abre aún un último capítulo antes de las conclusiones, en el considera la noción de dignidad, autonomía y libertad, donde realiza una muy profunda reflexión sobre 
estos términos, por ejemplo apostando por una acepción menos liberal y más republicana del término libertad, o señalando la importancia de un marco valorativo para dotar de sentido el concepto de dignidad.

Tras todo ello, la conclusión del autor es que, considerada la propuesta razonable de un mercado de órganos, y tratados sus argumentos de forma racional (es decir, sin apoyarse para rechazarlos en la repugnancia instintiva que pudiera seguirse de ellos, sino examinándolos con cuidado) hay razones tanto desde un enfoque individual como desde un enfoque social para considerar la venta de órganos una práctica de alto riesgo indeseable en la sociedad.

Concluyendo esta reseña, señalar tan solo que es una alegría leer una argumentación tan fundada y sólida en defensa de la dignidad humana más allá del precio. Esta obra se publica paralela en el tiempo a la última publicación de Jesús Ballesteros, otro catedrático de la misma área, ya emérito, donde aborda, desde otro enfoque y con otro estilo, una defensa similar. Ambos autores coinciden, cada uno desde su propio método, en la fundamental defensa de la dignidad humana frente al atropello al que pueden someterla los poderes (en este caso) económicos. Una convergencia semejante, realizada de formas tan paralelas, no puede sino sugerir la especial importancia que tiene, ahora y siempre, la defensa del carácter no mercantil de la persona. Defensa que en el caso de García Manrique se realiza de forma metódica, rigurosa, abierta y dialogante, ofreciendo un resultado llamativamente claro y fundamentado. 